\title{
A Five-Level Framework for Research on Process Mining
}

\author{
Jan vom Brocke $\cdot$ Mieke Jans $\cdot$ Jan Mendling $\cdot$ Hajo A. Reijers
}

Published online: 7 September 2021

(C) The Author(s) 2021

\section{Introduction}

Process Mining is a novel technology that helps enterprises to better understand their business processes. Over the last 20 years, intensive research has been conducted into various process mining techniques. These techniques support the automatic discovery of business process models from event $\log$ data, the checking of conformance between specified and observed behavior, the identification of various variants of a business process, non-compliant behavior, performance-relevant insights, and so forth.

Research on process mining has mostly focused on devising new or better algorithms (see van der Aalst 2016; Augusto et al. 2019a). There are a few exceptions, among others the following. van der Aalst et al. (2007) were the first to discuss process mining from the perspective of applications in industrial practice. Jans et al. (2014) applied process mining techniques to enrich audit evidence during a financial statement audit. vom Brocke and Mendling (2018) and vom Brocke et al. (2021) present various applications of process mining in hospitals, insurances, software usability analysis, and logistics.

J. vom Brocke

University of Liechtenstein, Vaduz, Liechtenstein

M. Jans

Hasselt University, Hasselt, Belgium

J. Mendling ( $\square)$

Humboldt-Universität zu Berlin, Berlin, Germany

e-mail: jan.mendling@hu-berlin.de

H. A. Reijers

Utrecht University, Utrecht, The Netherlands
In recent years, process mining has seen an increasing uptake in enterprises (Dumas et al. 2018), and has thus become an integral part of their daily business process management. Companies like Celonis, Fluxicon, Signavio, and Software AG are among the roughly 20 companies that Gartner monitors. As Kerremans (2019) from Gartner states, enterprises adopt process mining tools in order to support business process improvement, auditing and compliance, process automation, digital transformation, and IT operations (in order of decreasing importance).

Some contributions have been made towards understanding how process mining has an impact in an enterprise setting. Much of this research focuses on methodology and application domains. For instance, van Eck et al. (2015) and Aguirre et al. (2017) describe methodologies how process mining projects can be conducted, and Maruster and van Beest (2009) provide a methodology how business processes can be redesigned with the help of process mining. Mans et al. (2013) discuss success factors for such process mining projects. Examples of domain-specific proposals in healthcare are Rebuge and Ferreira (2012) and Fernández-Llatas et al. (2015). Thiede et al. (2018) find applications for digital as well as for physical processes, which are investigated using data from single systems, across systems, and across boundaries. Process mining has even been identified as a strategy of inquiry for studying organizational change (Grisold et al. 2020).

What is largely missing so far is research on how enterprises adopt process mining technology, how they integrate it into their information systems landscape, and which kind of effects emerge from this adoption. Effects are complex and unfold at different levels of the organization (Grisold et al. 2021). They are connected with organizational culture and the governance structures, to name but a few. Leonardi and Treem (2020) have coined 
the term behavioral visibility, a term that nicely emphasizes what process mining affords. The "datafication" of private and professional lives creates digital traces in various systems which can be analyzed by means of process mining techniques. In this way, process miming has the potential to afford behavioral visibility of various actions not only inside but also outside an organization. Obviously, many challenges arise from such large-scale behavioral visibility, including ethical ones. Therefore, more interdisciplinary research on the application of process mining from an enterprise perspective is needed.

In this editorial, we develop a framework for systematically discussing many of the associated concerns that emerge from adopting process mining in an enterprise setting. Our framework can be used to analyze the effects of process mining at different levels of investigation. In the following, we first provide a brief overview of process mining and its essential concepts. Then, we introduce our framework and discuss potential relevant research perspectives for each of its five levels.

\section{Techniques, Tasks and Parties Involved in Process Mining}

Enterprise information systems automatically $\log$ data during daily process executions. Process mining is a family of techniques that extract process knowledge from this logged process data. These techniques integrate concepts and ideas from machine learning and data mining on the one hand and process modeling and process analysis on the other hand (van der Aalst 2016).

In essence, process mining techniques support process discovery, conformance checking, process variant analysis, and process performance analysis (Dumas et al. 2018). Process discovery is the act of discovering a process model from event log data. This process model represents the real, observed behavior. Conformance checking focuses on the relation between a process model and the observed behavior (Carmona et al. 2018). Conformance checking techniques identify and measure the discrepancies between model and log. Researchers mainly use conformance checking to assure the quality of the discovered process model, i.e., to which extent this model accurately represents the logged behavior. In this context, the event log is taken as reference against which conformance is checked. Practitioners are more often interested in identifying which cases violate the behavior prescribed by the model. This means that the process model is taken as the norm to check conformance against. Process variant analysis addresses the question which variants of the process exist and which characteristics they are correlated with. Corresponding techniques build for instance on clustering and the analysis of factors. Process performance analysis is concerned with the analysis of time, costs, quality and flexibility of a business process based on event log data. In this way, measures can be identified to speed up the process, save costs, improve quality, and extend flexibility.

Technical research on process mining has primarily focused on process discovery and conformance checking. Different algorithms have been proposed for both tasks at hand. For process discovery, the Inductive Miner (Leemans et al. 2014), the Evolutionary Tree Miner (Buijs et al. 2014), the Split Miner (Augusto et al. 2019b) and the ILP miner (van Zelst et al. 2018) are examples of recent techniques. For conformance checking, techniques can be divided into three types of approaches. Some techniques rely on checking whether the observed behavior is compliant with a set of rules (e.g., Maggi et al. 2011). These rules function as a norm to check against, similar to controlling functions in organizations. Other techniques are based on the replay of the logged behavior on the process model (e.g., Rozinat and van der Aalst 2008). Finally, techniques based on alignments build on aligning the process executions with the closest path in the process model, which provides basis for calculating a notion of distance (e.g., De Leoni and van der Aalst 2013).

When organizations apply process mining, they do it by using a software tool from one of the numerous vendors. A process mining tool offers a set of analysis techniques for process analysts in a user-friendly way. The selection of the tool should reflect the requirements of the users. Often, these process mining users are process analysts who have the required skill set. Not only are they familiar with the field of process mining, but they also have expertise in an application domain. An experienced process analyst is a person who understands the organization's challenges, gets the right people on board, and is then capable of translating the business needs into specific analysis questions. Regarding process mining, process analysts have to develop an understanding which questions could be answered based on process event data. To this end, they interact with process participants, process stakeholders, and external partners. Process participants are those who work on individual tasks that collectively define overarching business processes. Their coordination and collaboration is logged by enterprise information systems, establishing the basis for applying process mining. Process stakeholders essentially include managers who have an interest in business processes operating well. They set the agenda for analyzing and improving business processes. Finally, system engineers provide expertise in which data enterprise information systems store and how event $\operatorname{logs}$ can be extracted.

A last, related party in the context of process mining is the group of external partners. These are the parties that 
are not directly involved in the process mining project, but are often considered in process analyses. The two most often analyzed business processes are order-to-cash and procure-to-pay. Both directly relate to external partners, namely customers and suppliers.

The described techniques, their corresponding analysis tasks, and the parties involved in process mining influence its success.

\section{A Framework for Research on Process Mining}

Process mining unfolds effects at different levels. For our framework we take Hevner et al. (2004) as a starting point, who describe a technical, a people and an organizational level of analysis. We refine this set to five levels, distinguishing an individual and a group level, and adding an ecosystem level (see Fig. 1).

At each level of the research framework, we identify specific phenomena of interest, key candidate theories to apply and further develop, and we pose a set of tangible research questions to be addressed as part of an agenda for future research. Please note that the separation of different levels is conceptual and, therefore, artificial. Even though effects span across these levels, the distinction of different levels can help to provide conceptual clarity.

\subsection{Technical Level}

Various concerns apply to researching process mining at the technical level. Much of the contributions at this level can be understood as pieces of engineering, and most of this engineering is focused on developing novel algorithms for different process mining tasks. These algorithms support the essential sets of various process mining techniques. Research on process mining at the technical level can be framed as a specific category of algorithm engineering.

Mendling et al. (2021) distinguish both design and knowledge contributions in the context of algorithm engineering:

Design contributions can be either design improvements or design exaptations. Design improvements present algorithms that perform better in at least one of the important performance dimensions such as execution time or output accuracy. For instance, the Split Miner (Augusto et al. 2019b) was presented as a design improvement providing high and balanced fitness and precision. Design exaptations demonstrate the applicability of established algorithmic designs for newly described tasks. An example is the work by van der Aa et al. (2018), which presents a conformance checking technique that is able to use text descriptions as normative specifications.

Knowledge contributions can be either performance propositions, sensitivity propositions, or explanatory propositions. The survey and comparison of state-of-the-art algorithms by Augusto et al. (2019a) focuses on performance propositions. Sensitivity propositions can be investigated with internal, design-related variations and external conditions as factors. The research by Di Ciccio et al. (2013), which studies the effect of noise on declarative process discovery, belongs to this category. Finally, explanatory propositions bring to the foreground the mechanisms of how design characteristics affect performance. For example, the study by Augusto et al. (2021), which investigates log complexity measures as predictors for the accuracy of process discovery, is in this category.
Fig.1 Process mining research framework

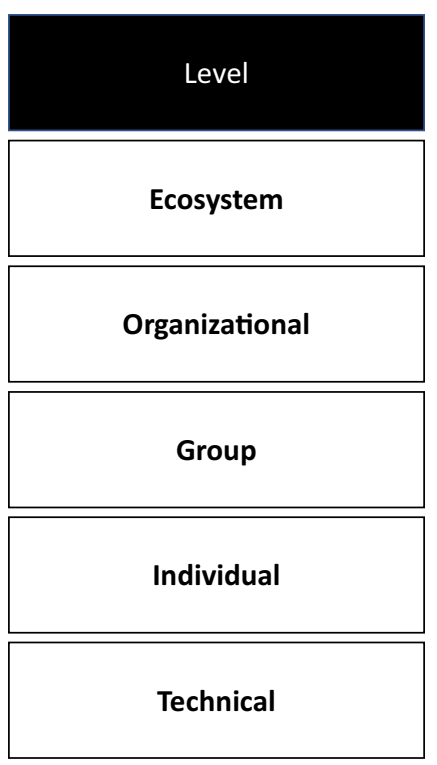


Much of the research on process mining at the technical level emphasizes design contributions and provides some knowledge contribution as an evaluation of the design work. Mendling et al. (2021) stress that various validity concerns have to be considered for such evaluations of process mining design contributions: algorithm engineering in general is subject to threats that relate to ecological validity, implementation validity, justification validity, logical validity, internal validity, external validity, construct validity, and conclusion validity.

\subsection{Individual Level}

Different categories of users work with process mining tools and their implemented algorithms and analysis techniques. We have identified users such as process analysts, process participants, process stakeholders, and external partners (Grisold et al. 2021). They use these tools in order to accomplish goals that are associated with process-mining-related tasks. Often, these tasks are not isolated, but embedded in BPM projects (Dumas et al. 2018) and BPM programs (vom Brocke et al. 2021). Some of the methodological specifics of these projects have been highlighted by van Eck et al. (2015), Aguirre et al. (2017), Maruster and van Beest (2009) and Mans et al. (2013), partially inspired by the CRISP-DM procedure (Martínez-Plumed et al. 2019). Ailenei et al. (2011) describe a set of 19 different analysis tasks including discovering the distribution of cases over paths, checking exceptions from the normal path, resources involved in cases, longest waiting times, identification of business rules. All of them can be directly supported by analysis based on process mining.

The task perspective plays a role for understanding why users adopt and use technology such as process mining tools. Seminal work towards the technology acceptance model emphasizes that perceptions about usefulness and ease of use are central for usage (Davis 1989; Davis et al. 1989). On the one hand, this is a question of how clear, understandable and easy to learn a technology is. On the other hand, different dimensions of usefulness such as job performance, work productivity, and overall effectiveness are equally important. Acceptance is indeed an issue for process mining (Grisold et al. 2021). According to the technology acceptance model, users are most likely to adopt process mining tools when they are easy to use and at the same time improve their effectiveness when working on process analysis tasks.

While the technology acceptance model explains when users are inclined to use a software tool, the task-technology fit model puts more emphasis on the actual task performance. Goodhue and Thompson (1995) stress that task characteristics and technology characteristics have to fit one another in order to provide a positive impact on performance. Applied to process mining, the fit model suggests that the analysis capabilities of a process mining tool should meet the demands of the tasks that a process analyst and other users are confronted with in the context of a BPM project. The tasks described by Ailenei et al. (2011) or the BPM use cases by van der Aalst (2013) could serve as basis for assessing such a fit.

Several additional perspectives on technology use have been integrated into the most recent version of the unified theory of acceptance and use of technology by Venkatesh et al. (2003, 2016). In essence, this theory posits that behavioural intentions are influenced by performance and effort expectancies, as well as social influence. These intentions materialize into actual technology usage under consideration of additional facilitating conditions. For process mining, social influence is a particularly interesting construct that can potentially play into different directions: from bottom up, it can produce resistance against creating transparency, eventually hampering adoption and use; from top down, social pressure can be imposed to make use of analysis capabilities of process mining. Such forces represent higher-level contextual factors (Venkatesh et al. 2016) that together with individual-level contextual factors influence acceptance, use, and eventually outcomes.

\subsection{Group Level}

We have described several groups of actors that are involved with business processes and corresponding BPM projects, namely process participants, process owners, process managers and process experts of multiple local teams. Notably, process participants and process managers are the largest and most diverse of these groups. A single business process can involve several departments and their corresponding managers and process participants who might not even be in the same reporting line. This setting provides various challenges for any initiative to improve such business processes (Markus and Jacobson 2015).

Before any improvements can be achieved, a shared understanding of the business process by all of the involved persons has to be established. In their work on the principles for good BPM, vom Brocke et al (2014) have formulated the principle of a joint understanding, meaning that BPM should not be the language of experts but create shared meaning. The BPM lifecycle addresses this point by stressing the need to discover and analyze the as-is process. Work on knowledge management in information systems research emphasizes this point, too. Nelson and Cooprider (1996) demonstrate that information system related activities require mutual trust and mutual influence, and that shared understanding and appreciation is key for translating mutual trust and influence into good performance. Process mining, in turn, might presumably help to increase 
both mutual trust and influence thanks to evidence-based insights into the process, as well as shared understanding by providing process representations that span the boundaries and the lines of visibility of the groups involved.

One of the relevant mechanisms for explaining the impact of process mining in this context are boundary objects. Star and Griesemer (1989) discuss cooperation without central control. They observe that boundary objects facilitate this cooperation thanks to three properties: interpretive flexibility, the needs of information and work processes, and dynamics of usage. Process mining tools can be analyzed using this lens, surfacing this facilitating role for the cooperation between, among others, process analysts, participants, and managers. The information needs of these groups differ such as the interpretations of representations generated by process mining tools, but they are not arbitrary. In this way, dynamic usage can converge towards standardized objects or systems (Star 2010), where boundary spanners-in-practice and boundary objects-in-use leverage cooperation (Levina and Vaast 2005).

Another relevant mechanism associated with process mining is behavioural visibility (Leonardi and Treem 2020). The digitalization of the work place has provided the means for tracking and analyzing behavior. An important observation regarding this digitalization is that the effort for obtaining behavior-related information has drastically declined as has the potential to analyze patterns (Leonardi and Treem 2020). Process mining tools leverage this behavioral visibility into work processes in organizations, revealing patterns, causes and motives (Leonardi and Treem 2020) by corresponding analysis functionality. In this way, new affordances and constraints (Norman 1999) are introduced into the way in which BPM projects are conducted. The article by Eggers et al. (2021) in this special issue discusses the mechanisms by which behavioral visibility increases process awareness, and eventually fosters process change.

We envision process mining in an enterprise setting to change the governance models for process management. Given the capacity to generate process knowledge quickly and continuously, based on real-time process data, process work will be less concerned with inquiring about processes and manually crafting processes models. Process mining will lead to more ad hoc investigations into processes and more real-time and data-driven decision making. Instead of working on processes in large teams of process analysts, investigations into processes could be organized in crossdepartmental meetings, e.g., held on a weekly basis and taking immediate action. Hence, process mining also stimulates research on the organization of the process work.

\subsection{Organization Level}

Technical implementation, individual adoption, and actual use of process mining tools are a prerequisite for any impact at the level of organizational performance. The mechanisms at the group level reveal how process mining can unfold its impact at the level of the larger organization. The information systems success model makes exactly this point by highlighting the impact of system quality, information quality, and service quality on individual use and usage satisfaction; these eventually translate into net benefits at the individual and at the organizational level (DeLone and McLean 1992, 2003; Petter et al. 2008).

The theory of effective use drills down into the mechanisms surrounding information quality. In essence, effective use builds on a chain of transparent interaction, representational fidelity and informed action, which all contribute to efficient and effective performance (BurtonJones and Grange 2013). Trieu et al. (2022) contextualize effective use in a business intelligence context and foreground business intelligence system quality, data integration, and an evidence-based management culture. For process mining, these constructs might serve as potential constraints to the affordances a process mining tool provides.

What is partially hidden behind the service quality construct in the success model is a capability perspective. BPM-related capabilities have often been described as dynamic capabilities, which are directed towards organizational problem solving (Niehaves et al. 2014). The BPMrelated capability areas presented by Rosemann and vom Brocke (2015) are specifically relevant in this context. The Delphi study by Martin et al. (2021) in this special issue uses them as a framework for identifying challenges and opportunities arising from process mining. The experts in this study describe more opportunities related to strategic alignment, methods and information technology, while more challenges are identified for governance, people and culture. Also in this special issue, Eggers et al. (2021) emphasize that the benefits that process mining offers are contingent to governance and implementation approaches.

Process mining can also be understood as a specific big data analytics capability. The framework by Grover et al. (2018) offers insights into how such capabilities along with an underlying infrastructure unfold an impact in different value dimensions. They describe that different value creation mechanisms are key to the capability realization process, including organization performance, business process improvement, product and service innovation, and consumer experience as much as market enhancement (Grover et al. 2018). Finally, Grover et al. (2018) point to various other theoretical logics that can be useful for studying big data analytics, namely resources, alignment, 
real options, dynamics, and absorptive capacity. These might be equally relevant for process mining.

\subsection{Digital Ecosystem Level}

So far, process mining has largely been restricted to the boundaries of central organizations. Martin et al. (2021) identify opportunities and challenges for process mining, and several of these directly relate to the ecosystem in which a company operates. The opportunities described by experts of their Delphi study relate to how process mining can facilitate value creation by fostering collaboration across organizational boundaries.

At this point, some research has been conducted on how process mining can be implemented at an inter-organizational level. Before organizational and strategic challenges can be addressed, various conceptual challenges have been overcome for constructing an integrated coherent data representation of the process across involved organizations (Gerke et al. 2009; Dumas et al. 2018, Chapter 11). Opportunities arise from the increasing uptake of blockchain technology for business processes (Mendling et al. 2018; Pufahl et al. 2021). Specific technical solutions such as the extraction of blockchain data for processes have been devised (e.g., Klinkmüller et al. 2019; Mühlberger et al. 2019). Hobeck et al. (2021) demonstrate which kind of insights can be derived by help of their case study with Augur.

Grover emphasizes in his interview with Mendling and Jans (2021) in this special issue that "the digital" defines new challenges for researching business processes. In this context, also new challenges arise. For instance, privacy is a concern once data is analyzed that is related to people who are not part of the same organization as the one in which the data is analyzed or where the generated insights are used (see Mannhardt et al. 2019). This is particularly relevant for mining data from the Internet of Things (Michael et al. 2019) and applications in healthcare (Pika et al. 2020).

\section{Future Research Directions}

In this editorial, we have identified connections between process mining and many established concepts and theories on information systems. We described a five-level framework including a technical, individual, group, organization, and ecosystem level. The impact of process mining can be investigated at each of these levels and across them.

In our call for papers for this special issue, we raised several research questions (vom Brocke et al. 2020a, b):
- How is process mining used and adopted at the enterprise level?

- What is the potential of using various types of data in process mining?

- How does process mining complement other approaches and technologies?

- How do enterprises build suitable data sets?

- What are the implications for management of using process mining?

- Which governance structures do enterprises develop for process mining?

- How do enterprises calculate the business case of process mining?

- How does process mining change organizational culture?

- How does process mining change the required skill sets of tool users?

- How is process mining integrated into the IT landscape?

- How is process mining integrated with existing business process methodologies?

- How is process mining adopted in specific application domains, e.g., accounting, health, finance, HR, tax, etc.?

- How is process mining used to support digital transformation initiatives?

- What strategic implications for enterprises emerge from process mining usage?

- What is the business impact of adopting process mining?

- What is the overall business value of process mining?

- What is the transformative nature of process mining at the enterprise level?

The two research articles (Eggers et al. 2021; Martin et al. 2021) and the interview (Mendling and Jans 2021) published in this special issue answer some of these questions. Many of the questions, however, remain open.

The process mining research framework also shows that contributions from different disciplines are needed to further understand and develop the potential of process mining. On a technical level, for instance, computer science makes important contributions to algorithm engineering. Information systems research, in addition, has a great opportunity to cover the many socio-technical aspects related to process mining use on the individual, group, organizational and ecosystem level.

Specifically, both behavioral and design-oriented contributions are needed (Hevner et al. 2004). Based on a better understanding of process mining use in an enterprise setting, prescriptive knowledge can be gained to support interventions in practice (vom Brocke et al. 2020a, b), e.g., by models and methods for value identification and value 
realization through process mining. We hope that this special issue will trigger a range of research activities to address many of these research questions.

Funding Open Access funding enabled and organized by Projekt DEAL.

Open Access This article is licensed under a Creative Commons Attribution 4.0 International License, which permits use, sharing, adaptation, distribution and reproduction in any medium or format, as long as you give appropriate credit to the original author(s) and the source, provide a link to the Creative Commons licence, and indicate if changes were made. The images or other third party material in this article are included in the article's Creative Commons licence, unless indicated otherwise in a credit line to the material. If material is not included in the article's Creative Commons licence and your intended use is not permitted by statutory regulation or exceeds the permitted use, you will need to obtain permission directly from the copyright holder. To view a copy of this licence, visit http://creativecommons. org/licenses/by/4.0/.

\section{References}

Aguirre S, Parra C, Sepúlveda M (2017) Methodological proposal for process mining projects. Int $\mathrm{J}$ Bus Process Integr Manag $8(2): 102-113$

Ailenei I, Rozinat A, Eckert A, van der Aalst WM (2011) Definition and validation of process mining use cases. In: International conference on business process management. Springer, Heidelberg, pp 75-86

Augusto A, Conforti R, Dumas M, La Rosa M, Maggi FM, Marrella A, Mecella M, Soo A (2019a) Automated discovery of process models from event logs: review and benchmark. IEEE Trans Knowl Data Eng 31(4):686-705

Augusto A, Conforti R, Dumas M, La Rosa M, Polyvyanyy A (2019b) Split miner: automated discovery of accurate and simple business process models from event logs. Knowl Inf Syst 59(2):251-284

Augusto A, Mendling J, Vidgof M, Wurm B (2021) The connection between process complexity of event sequences and models discovered by process mining. arXiv preprint arXiv:2106.07990

Buijs JC, van Dongen BF, van der Aalst WM (2014) Quality dimensions in process discovery: the importance of fitness, precision, generalization and simplicity. Int J Coop Inf Syst 23(01): 1440001

Burton-Jones A, Grange C (2013) From use to effective use: a representation theory perspective. Inf Syst Res 24(3):632-658

Carmona J, van Dongen B, Solti A, Weidlich M (2018) Conformance checking. Springer, Cham

Davis FD (1989) Perceived usefulness, perceived ease of use, and user acceptance of information technology. MIS Q 13:319-340

Davis FD, Bagozzi RP, Warshaw PR (1989) User acceptance of computer technology: a comparison of two theoretical models. Manag Sci 35(8):982-1003

De Leoni M, Van Der Aalst WM (2013) Aligning event logs and process models for multi-perspective conformance checking: an approach based on integer linear programming. In: Daniel $\mathrm{F}$, Wang J, Weber B (eds) Business process management. Springer, Heidelberg, pp 113-129

DeLone WH, McLean ER (1992) Information systems success: the quest for the dependent variable. Inf Syst Res 3(1):60-95
DeLone WH, McLean ER (2003) The DeLone and McLean model of information systems success: a 10-year update. J Manag Inf Syst 19(4):9-30

Di Ciccio C, Mecella M, Mendling J (2013) The effect of noise on mined declarative constraints. In: International symposium on data-driven process discovery and analysis. Springer, Heidelberg, pp 1-24

Dumas M, La Rosa M, Mendling J, Reijers HA (2018) Fundamentals of business process management, 2nd edn. Springer, Heidelberg

Eggers J, Hein A, Böhm M, Krcmar H (2021) No longer out of sight, no longer out of mind? How organizations engage with process mining-induced transparency to achieve increased process awareness. Bus Inf Syst Eng 63(5). https://doi.org/10.1007/ s12599-021-00715-x

Fernández-Llatas C, Lizondo A, Monton E, Benedí J-M, Traver V (2015) Process mining methodology for health process tracking using real-time indoor location systems. Sensors 15(12):29821-29840

Gerke K, Claus A, Mendling J (2009) Process mining of RFID-based supply chains. In: 2009 IEEE Conference on commerce and enterprise computing. IEEE, pp 285-292

Goodhue DL, Thompson RL (1995) Task-technology fit and individual performance. MIS Q 19:213-236

Grisold T, Wurm B, Mendling J, vom Brocke J (2020) Using process mining to support theorizing about change in organizations. In: Proceedings HICSS 2020

Grisold T, Mendling J, Otto M, vom Brocke J (2021) Adoption, use and management of process mining in practice. Bus Process Manag J 27(2):369-387

Grover V, Chiang RH, Liang TP, Zhang D (2018) Creating strategic business value from big data analytics: a research framework. J Manag Inf Syst 35(2):388-423

Hevner AR, March ST, Park J, Ram S (2004) Design science in information systems research. MIS Q 28:75-105

Hobeck R, Klinkmüller C, Bandara H. D, Weber I, Van der Aalst W (2021) Process mining on blockchain data: a case study of Augur. In: International conference on business process management

Jans M, Alles MG, Vasarhelyi MA (2014) A field study on the use of process mining of event logs as an analytical procedure in auditing. Account Rev 89(5):1751-1773

Kerremans M (2019) Market guide for process mining. Gartner Report

Klinkmüller C, Ponomarev A, Tran AB, Weber I, van der Aalst W (2019) Mining blockchain processes: extracting process mining data from blockchain applications. In: International conference on business process management. Springer, Cham, pp 71-86

Leemans SJ, Fahland D, Van Der Aalst WM (2014) Process and deviation exploration with inductive visual miner. BPM (demos) 1295(8):46

Leonardi PM, Treem JW (2020) Behavioral visibility: a new paradigm for organization studies in the age of digitization, digitalization, and datafication. Org Stud 41(12):1601-1625

Levina N, Vaast E (2005) The emergence of boundary spanning competence in practice: implications for implementation and use of information systems. MIS Q 29:335-363

Maggi FM, Montali M, Westergaard M, van der Aalst WMP (2011) Monitoring business constraints with linear temporal logic: an approach based on colored automata. In: International conference on business process management. Springer, Heidelberg, pp 132-147

Mannhardt F, Koschmider A, Baracaldo N, Weidlich M, Michael J (2019) Privacy-preserving process mining. Bus Inf Syst Eng 61(5):595-614 
Mans R, Reijers HA, Berends H, Bandara W, Prince R (2013) Business process mining success. In: Proceedings ECIS 2013, pp 89ff

Markus ML, Jacobson DD (2015) The governance of business processes. In: vom Brocke J, Rosemann M (eds) Handbook on business process management, volume 2: strategic alignment, governance, people and culture, 2nd edn. Springer, Berlin

Martin N, Fischer D, Kerpedzhiev G, Goel K, Leemans S, Röglinger M, van der Aalst WMP, Dumas M, La Rosa M, Wynn M (2021) Opportunities and challenges for process mining in organisations - results of a Delphi study. Bus Inf Syst Eng 63(5) (in print)

Martínez-Plumed F, Contreras-Ochando L, Ferri C, Orallo JH, Kull M, Lachiche N, Ramírez-Quintana MJ, Flach PA (2019) CRISPDM twenty years later: from data mining processes to data science trajectories. IEEE Trans Knowl Data Eng 33(8):3048-3061. https://doi.org/10.1109/TKDE.2019.2962680

Maruster L, van Beest NRTP (2009) Redesigning business processes: a methodology based on simulation and process mining techniques. Knowl Inf Syst 21(3):267-297

Mendling J, Jans M (2021) Interview with Varun Grover on business processes, information technology and its evolution in the digital age. Bus Inf Syst Eng 63(5):1-4

Mendling J, Weber I, van der Aalst W, Brocke JV, Cabanillas C, Daniel F, Debois S, Di Ciccio C, Dumas M, Dustdar S, Gal A, García-Bañuelos L, Governatori G, Hull R, La Rosa M, Leopold H, Leymann F, Recker J, Reichert M, Reijers HA, Rinderle-Ma S, Solti A, Rosemann M, Schulte S, Singh MP, Slaats T, Staples M, Weber B, Weidlich M, Weske M, Xu X, Zhu L (2018) Blockchains for business process management-challenges and opportunities. ACM Trans Manag Inf Syst 9(1):1-16

Mendling J, Depaire B, Leopold H (2021) Theory and practice of algorithm engineering. arXiv preprint arXiv:2107.10675

Michael J, Koschmider A, Mannhardt F, Baracaldo N, Rumpe B (2019) User-centered and privacy-driven process mining system design for IoT. In: International conference on advanced information systems engineering. Springer, Cham, pp 194-206

Mühlberger R, Bachhofner S, Di Ciccio C, García-Bañuelos L, López-Pintado O (2019) Extracting event logs for process mining from data stored on the blockchain. In: International conference on business process management. Springer, Cham, pp 690-703

Nelson KM, Cooprider JG (1996) The contribution of shared knowledge to IS group performance. MIS Q 20(4):409-432

Niehaves B, Poeppelbuss J, Plattfaut R, Becker J (2014) BPM capability development - a matter of contingencies. Bus Process Manag J 20(1):90-106. https://doi.org/10.1108/BPMJ-07-20120068

Norman DA (1999) Affordance, conventions, and design. Interactions 6(3):38-43

Petter S, DeLone W, McLean E (2008) Measuring information systems success: models, dimensions, measures, and interrelationships. Eur J Inf Syst 17(3):236-263

Pika A, Wynn MT, Budiono S, Ter Hofstede AH, van der Aalst WM, Reijers HA (2020) Privacy-preserving process mining in healthcare. Int J Environ Res Public Health 17(5):1612

Pufahl L, Ohlsson B, Weber I, Harper G, Weston E (2021) Enabling financing in agricultural supply chains through blockchain. In: Vom Brocke J, Mendling J (eds) Business process management cases, vol 2. Springer, Heidelberg, pp 41-56

Rebuge A, Ferreira DR (2012) Business process analysis in healthcare environments: a methodology based on process mining. Inf Syst 37(2):99-116
Rosemann M, vom Brocke J (2015) The six core elements of business process management. In: vom Brocke J, Rosemann M (eds) Handbook on business process management, vol 1. Springer, Heidelberg, pp 105-122

Rozinat A, van der Aalst WM (2008) Conformance checking of processes based on monitoring real behavior. Inf Syst 33(1):64-95

Star SL (2010) This is not a boundary object: reflections on the origin of a concept. Sci Technol Hum Values 35(5):601-617

Star SL, Griesemer JR (1989) Institutional ecology, translations' and boundary objects: amateurs and professionals in Berkeley's Museum of Vertebrate Zoology, 1907-39. Soc Stud Sci 19(3):387-420

Thiede M, Fürstenau D, Barquet APB (2018) How is process mining technology used by organizations? A systematic literature review of empirical studies. Bus Process Manag J 24(4):900-922. https://doi.org/10.1108/BPMJ-06-2017-0148

Trieu V-H, Burton-Jones A, Green P, Cockcroft S (2022) Applying and extending the theory of effective use in a business intelligence context. MIS Q 46:forthcoming

van der Aalst WM (2013) Business process management: a comprehensive survey. Int Sch Res Not. https://doi.org/10.1155/2013/ 507984

van der Aalst WMP (2016) Process mining - data science in action, 2nd edn. Springer, Heidelberg

van der Aa H, Leopold H, Reijers HA (2018) Checking process compliance against natural language specifications using behavioral spaces. Inf Syst 78:83-95

van der Aalst WMP, Reijers HA, Weijters AJMM, van Dongen BF, Alves de Medeiros AK, Song M, Verbeek HMW (2007) Business process mining: an industrial application. Inf Syst 32(5):713-732

van Eck ML, Lu X, Leemans SJJ, van der Aalst WMP (2015) PM²: a process mining project methodology. Proc CAiSE 2015:297-313

van Zelst SJ, van Dongen BF, van der Aalst WM, Verbeek HMW (2018) Discovering workflow nets using integer linear programming. Computing 100(5):529-556

Venkatesh V, Morris MG, Davis GB, Davis FD (2003) User acceptance of information technology: toward a unified view. MIS Q 27(3):425-478

Venkatesh V, Thong JY, Xu X (2016) Unified theory of acceptance and use of technology: a synthesis and the road ahead. J Assoc Inf Syst 17(5):328-376

vom Brocke J, Mendling J (2018) Business process management cases. Digital innovation and business transformation in practice. Springer, Heidelberg

vom Brocke J, Schmiedel T, Recker J, Trkman P, Mertens W, Viaene S (2014) Ten principles of good business process management. Bus Process Manag J 20(4):530-548

vom Brocke J, Jans M, Mendling J, Reijers HA (2020a) Call for papers, issue 5/2021. Bus Inf Syst Eng 62(2):185-187

vom Brocke J, Winter R, Hevner A, Maedche A (2020b) Accumulation and evolution of design knowledge in design science research - a journey through time and space. J Assoc Inf Syst 21(3):520-544

vom Brocke J, Mendling J, Rosemann M (2021) Business process management cases, volume 2. Digital transformation, strategy, processes and execution. Springer, Heidelberg 